\title{
EVALUATION OF PRESSURE DROP IN FLOW OVER FIXED POROUS BED
}

\begin{abstract}
R. B. Cruz, ABSTRACT
D. R. S Guerra,

and T. L. Azevedo

Universidade Federal do Pará

Faculdade de Engenharia Mecânica

CEP. 66075-110, Belém, Pará, Brasil

daguerra@ufpa.br

Received: October 25, 2013

Many studies are conducted about the dynamics of fluids in porous media, which generates a number of factors and problems are solved. In particular the phenomenon of pressure drop in flows on fixed bed, although fairly well in the form Ergun's equation, still has certain inconsistencies with regard to the types of materials to be used in the packaging of the beds. The objective of this work is to study this phenomenon using some experiments reported in the literature to determine the pressure drop in fixed bed consisting of porous particles of açaí seed. Experimental studies were conducted to predict, and take into account the losses resulting from friction and inertia that showed strong dependence on velocity. The method of using the particular friction factor prediction as a way to replace the usual calculation and measurement of pressure drop. The analysis in the wind tunnel was made from different sizes of bed, with a decrease in arithmetic progressions corresponding to half the value of the diameter of the tube used in the test. Several bands of Reynolds number were also employed in order to be able to visualize how the phenomenon behaves on various tracks. For such a survey was necessary some parameters such as açaí seeds diameters, seed weight and volume occupied by the bed; these parameters are of vital importance in Ergun equation, because an important aspect of the phenomenon is the porosity which enters as a foundation in the theory of flow fixed bed. At the end of the study was found the divergence in big bands of the Reynolds number of the correlation between experimental data and the Ergun equation.
\end{abstract}

Revised: November 21, 2013

Accepted: December 20, 2013

Keywords: pressure drop, fixed bed, bed porosity, açaí seeds.

\section{NOMENCLATURE}

$\mathrm{A}_{\mathrm{p}} \quad$ Surface area of a single non-spherical particle,

$\mathrm{m}^{2}$

$\mathrm{A}_{\mathrm{sp}} \quad$ surface area of the equivalent-volume sphere,

$\mathrm{m}^{2}$

D Pipe inner diameter, $\mathrm{m}$

$\mathrm{D}_{\mathrm{p}} \quad$ Particle Diameter, $\mathrm{m}$

$\mathrm{f}_{\mathrm{p}} \quad$ Particle Friction Factor

$\mathrm{L} \quad$ Bed Length, $\mathrm{m}$

$\mathrm{M}_{\text {bed }}$ Bed Mass, $\mathrm{g}$

$\mathrm{m}_{\text {seed }}$ Particle Mass, g

$\mathrm{Re}_{\mathrm{p}} \quad$ Reynolds number based of particle

$\mathrm{U}$ Superficial velocity, $\mathrm{m} / \mathrm{s}$

$\mathrm{V}_{\text {bed }}$ Bed Volume, $\mathrm{m}^{3}$

$\mathrm{V}_{\text {empty }}$ Volume of Void, $\mathrm{m}^{3}$

$\mathrm{V}_{\mathrm{p}} \quad$ Volume of a single non-spherical particle, $\mathrm{m}^{3}$

$\mathrm{V}_{\text {reactor }}$ Volume of reactor, $\mathrm{m}^{3}$

$\mathrm{x} \quad$ Cartesian axis direction, $\mathrm{m}$

y Cartesian axis direction, $\mathrm{m}$

$\mathrm{z} \quad$ Cartesian axis direction, $\mathrm{m}$

\section{Greek symbols}

$\varepsilon \quad$ porosity

$\rho \quad$ density, $\mathrm{kg} / \mathrm{m}^{3}$ $\begin{array}{ll}\mu & \text { viscosity dynamic, } \mathrm{m}^{2} / \mathrm{s} \\ \psi & \text { sphercity }\end{array}$

\section{INTRODUCTION}

In the fluid dynamics over porous beds many studies are focused on different applications including flow over fixed bed which we can draw important information, among them the application of these beds in the gasification phenomenon. How important is determining the pressure drop caused by the bed is the target of study in the first instance the data base search with equations conductors in the literature. In the overview regional, the gasification process comes to using native Amazon biomass that is the açaí seed to develop the studied on the art of gasification.

The wide application of fixed beds in the engineering field, in particular its use in gasifiers reactors makes the study of fluid flow and the previous pressure drop diagnostic through this kind of equipment becomes indispensable. The relevance of the pressure loss determination provide a broad overview how the flow behaves and how it influences on the gasifiers performance; despite of that, it is important to investigate the reactor performance according to the biomass physical characteristics and 
the more appropriate operational mode to improve the process.

On the construction and development of new technologies for gasifiers, the operational parameters still are the great challenges which require close attention, so that we can improve them and determine the best operational conditions. An observed difficulty is concerned to the pressure drop caused by the fixed porous bed. The height of the porous medium can becomes impracticable the process or to reduce substantially the reactor performance. An estimative or a determination of the pressure drop caused by the porous bed in order to have knowledge of the phenomena will enable to improve the process so that it will not be an obstacle to the correct functioning of all equipments connected to the gasifier reactor.

A vast amount of information in the form of empirical and semi-empirical correlations which relate the pressure drop to the hydrodynamic conditions of the packed beds is available held a correspondence with the equation that governs this type of flow, widely known as Ergun equation. The Ergun correlation (1952), has been widely used for design or treatment of raw experimental data extracted from certain geometrical configurations. Ergun's correlation accounts for viscous and inertial energy losses and relates them to the dynamic variable, velocity of the fluid, as well as the structure of the bed, as characterized by the bed mean voidage.

Researches check the values of the empirical constants 150 and 1.75 from Ergun (1952) equation. A variety of empirical values have been encountered because of the use of different packaging materials (Montillet et al. 2007) such as spherical particles of regular shape or non-spherical particles of irregular shape. Universal values of the Ergun constants have been the subject of considerable speculation since 1952. The values of empirical constants in Ergun's correlation have been proposed; Leva (1947) proposed the 200 and 1.75, and Macdonald (1979) the 180 and $1.8-4.0$ as cited by Niven (2002). The rationale explication for the variation in the constants was determined as the changes in regular spherical particle shape for nonspherical particles. This explication leads to a theory held by most researchers that is satisfied with the fact that the values of the Ergun constants must be determined empirically for each bed. Such theory stems a belief that values are not only dependent on the geometry of the particle, but in addition they can vary from bed to bed macroscopic (made of the same particles) due to different structures of the pack in the bed after repackaging.

Macdonald et al. (1979) compared two friction factor-Reynolds number correlations, the nondimensional Forchheimer (1901) equation of Ahmed and Sunada (1969) and the modified Ergun equation, were tested statistically with a large number of experimental data. It is concluded that the physical basis of the Forchheimer (1901) equation appears to be accurate. The modified Ergun equation, while certainly not rigorous, can be expected to predict experimental results for unconsolidated media with an accuracy of $\pm 50 \%$. For the wide porosity range from 0.36 to 0.92 , the porosity function of the Ergun equation is superior to others proposed in the literature, although others are better over narrower porosity ranges. For nonspherical particles, it is necessary to measure the surface-to-volume ratio or the sphericity for use in $D_{p}$. These experimental studies were conducted to predict, being taken into account the losses resulting from friction and inertia that showed strongly dependent on velocity.

Macdonald et al. (1979), Churchill (1988); Kaviany (1995) used successfully Forchheimer (1901) Equation and correlating the data separately for different porous beds in terms of various parameters including the surface roughness of the particle.

Keyser et al. (2006) has measured pressure drop and flow distribution of gas through packed carbon layers. This knowledge is fundamental helpful in better understanding the operational performance of fixed bed gasifiers dry bottom. The results showed a general observation that is the known Ergun (1952) equation for the pressure drop of fluid through the packed bed not suitable if the size distribution of coal becomes too large. In this case, the decrease of actual pressure exceeds the pressure drop provided by the Ergun equation (1952).

$\mathrm{Li}$ and $\mathrm{Ma}$ (2011) carried out experimental studies to determine the characteristics of friction and pressure drop of the fluid flow in beds packed with spherical porous particles and non-spherical. The aim is to examine the applicability of the Ergun equation flow resistance rating is packed beds of non-spherical particles. They found that the pressure drops in the packed beds with hollow spheres and cylindrical particles are much higher than the predictions of the Ergun equation if the diameters of the spheres and cylinders are employed in the equation. The other models applying variations of the Ergun equation using Sauter mean particle diameter and modifying the Ergun constants, could not predict the pressure drops measured in their study.

The experimental results are analyzed in order to consider the analytical study of the pressure drop in porous media according to the Ergun equation (1952), which has long governed this type of flow and shows up on the optics of some studies, the primary point to analyze this type of flow. For the analysis of the porous bed, were raised as important factors: the porosity, the apparent velocity through the bed under the law of Darcy and pressure drop caused by the fixed bed. The methodology used for this study and the results are presented. Therefore will be seen as such a formulation does not satisfactorily meet all operating regimes to be put in the gasifier, it is necessary to investigate a more 
succinct about their real consequences.

For the analysis of porous bed important factors should be raised as: porosity of bed, sphericity of particle and pressure drop caused by the fixed bed. To determine the bed porosity data were collected from a sample of diameters of açaí seeds, and taken their average value, as well as its specific gravity by weighting each seed and doing general survey to determine the average of these, always obeying the variation of the standard deviation caused by the total samples. In this study experiments were performed with a fixed bed of açaí seed attached to a wind tunnel.

The actual study presents experimentally the pressure loss and to evaluate the pressure difference in porous medium formed by açaí seed and to determine the bed porosity using equations proposed by Ergun. The experimental study was carried out in a open wind tunnel with low turbulence; the used test section is similar to that used to the gasifier.

\section{POROSITY}

Porosity can be described as the dead space in a way which crosses a stream, and this would mean the fraction of empty space in relation to the total space in the porous medium occupies (Nield and Bejan, 1992). When porosity is closer to 1 would result in a reactor completely open without fills, as shown in Figure (1).

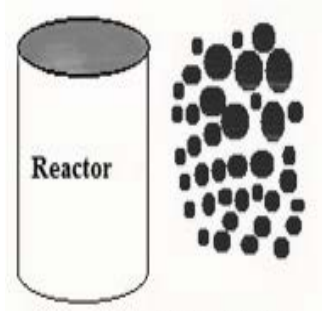

Açai's Seeds

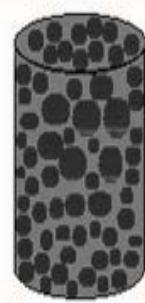

Porous Bed

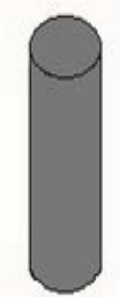

Empty Volume
Figure 1. Layout of porous bed.

The definition of equivalent particle diameter is based on the shape factor $(\psi)$ ( $\mathrm{Li}$ and $\mathrm{Ma}, 2011)$.

$$
\psi=\frac{\mathrm{Asp}}{\mathrm{Ap}_{\mathrm{p}}}=\frac{\pi^{1 / 3}\left(6 \mathrm{~V}_{\mathrm{p}}\right)^{2 / 3}}{\mathrm{Ap}_{\mathrm{p}}}
$$

The shape factor of the friction laws explicitly reflects the physical mechanism for the increased contribution of frictional drag due to the tortuous complex forms, particularly in the turbulent regime (Niven, 2000). Where $V_{p}$ is volume of the particle, $A_{p}$ the surface area of the particle, and $A_{s p}$ the surface area of the equivalent-volume sphere.

$$
\mathrm{D}_{\mathrm{vs}}=\frac{6 \mathrm{~V}_{\mathrm{p}}}{\mathrm{Asp}}=\frac{6 \mathrm{~V}_{\mathrm{p}}}{\psi \mathrm{Ap}_{\mathrm{p}}}
$$

The particle diameter defined in Eq.(2) is equal to the volume-surface mean diameter as referred in the literature (Ozahi et al. 2008).

To study the porosity it has been taken possession of the values obtained from measurements of the average diameter and average mass of the açaí seeds according to statistical described below.

The weight of each açaí stone was obtained by grouping all the values in a sample of 100 seeds weighing up to average, and always checking the standard deviation fit within a limit below 0.15 for the sample group. The measurements were performed on a sample of 230 açaí seeds and represent by the three diameters of the core, according to direction in Fig. (2).
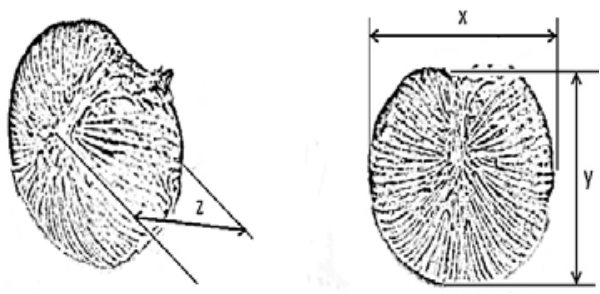

Figure 2. Three directions of measures for the diameters from açaí seed.

The sample that could determine the average diameter of açaí seeds is concentrated in a population of 100 açaí seeds in order to obtain a small standard deviation and to minimize the uncertainty of the measurements. Such mean values can be observed according to what is shown in Table 1.

Table 1. Mean diameters of the $\mathrm{X}, \mathrm{Y}$ and $\mathrm{Z}$ directions of açaí seeds.

\begin{tabular}{|c|c|c|c|}
\hline Directions & $\mathrm{X}$ & $\mathrm{Y}$ & $\mathrm{Z}$ \\
\hline $\begin{array}{c}\text { Average } \\
\text { Diameter (cm) }\end{array}$ & 1.16 & 0.98 & 0.97 \\
\hline $\begin{array}{c}\text { Standard } \\
\text { deviation }\end{array}$ & 0.069 & 0.084 & 0.065 \\
\hline
\end{tabular}

The porosity determination requires the diameter and mass averages of the açaí seed. So to weight açaí seed an analytical balance OHAUS model AL5000 with precision of $0.0001 \mathrm{~g}$, was used. The diameter seed was measured with a caliper of $0.05 \mathrm{~mm}$ in precision.

On calculation purposes the assumption that the seed of açaí is a perfect sphere was made, so we take an average for the three directions $\mathrm{X}, \mathrm{Y}$ and $\mathrm{Z}$ to getting an average value of the average diameter of açaí seeds as shown on Table 2 .

Table 2. Average diameter and volume of açaí seeds.

\begin{tabular}{|c|c|}
\hline Diameter $(\mathrm{cm})$ & 1.032 \\
\hline Uncertainty & \pm 0.01 \\
\hline Volume $\left(\mathrm{m}^{3}\right)$ & 0.57 \\
\hline
\end{tabular}


To study the porosity of the açaí bed was necessary to carried out an experiment to find out the volume of voids left in bed.

$$
\begin{gathered}
\mathrm{V}_{\text {bed }}=\mathrm{V}_{\text {seed }}\left(\frac{\mathrm{M}_{\text {bed }}}{\mathrm{m}_{\text {seed }}}\right) \\
\mathrm{V}_{\text {empty }}=\mathrm{V}_{\text {reactor }}-\mathrm{V}_{\text {bed }}
\end{gathered}
$$

The determination of the amount of space that is left blank relating to this is the volume of a core nucleus of açaí ( $\left.\mathrm{V}_{\text {seed }}\right)$, the relationship between the seed mass $\left(\mathrm{m}_{\text {seed }}\right)$ and the weight of bed $\left(\mathrm{M}_{\text {bed }}\right)$, is provided by Eq. (3).

The volume occupied by air, or air voids, is determined by the difference between the volume occupied by the açaí seeds and the total volume of the reactor, according to Eq. (4).

$$
\varepsilon=\frac{\mathrm{V}_{\text {empty }}}{\mathrm{V}_{\text {reactor }}}
$$

As it is the porosity of the void fraction by the total volume occupied, we have the Eq. (5).

Statistical analysis was performed for the data family obtained in this study; mean results and standard deviation are shown in Table 3.

Table 3. Statistical average weight of açaí seed.

\begin{tabular}{|c|c|}
\hline Average mass [g] & 0.69 \\
\hline Standard deviation & 0.141 \\
\hline Uncertainty [g] & \pm 0.02 \\
\hline
\end{tabular}

Whereas the particle açaí seed is not porous, the porosity is given solely by the set which is provided by the bed, $\rho$ is the density, given by the ratio between the mass and volume of the particle in isolation, value being found of $1,206 \mathrm{~kg} / \mathrm{m}^{3}$.

$$
\mathrm{M}_{\text {bed }}=(13.136 \mathrm{~L})-0.855
$$

Plotting the equation related to the different positions in the weight PVC pipe, through the data set it is possible to obtain the Eq. (6).

The void volume as determined above is the ratio of the total mass of the cores in the bed by açaí density.

The volume occupied by the açaí seeds and air voids caused by these were determined through mathematical relationships presented , the calculation to analyze the evolution of porosity was performed for various lengths of bed used in the experiment carried out in the wind tunnel.

Figure 3 shows a curve with a steep slope at the beginning and then increases as the bed length increases; after that the curve behavior presents a stabilization so as to form a line which leads us to conclude that the porosity remains stable after region from 2D.

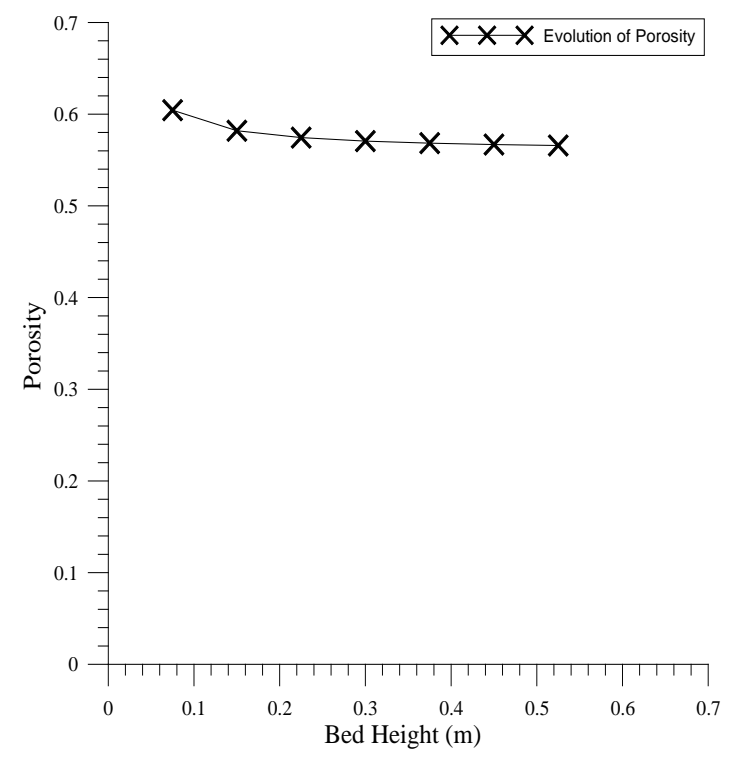

Figure 3. Evolution of porosity.

\section{ANALYTICAL SOLUTION OF THE ERGUN'S EQUATION}

The most commonly used to determine loss in porous beds is the law of Darcy. In which found that for laminar flow rate is proportional to the pressure drop $(\Delta \mathrm{P})$ and inversely proportional to the viscosity $(\mu)$ and the length $(L)$.

The most widespread for the general case is turbulent and laminar the semi empirical Ergun equation, where the first term of the equation is predominant for laminar flow, while the second is of greater importance for higher values of Reynolds, due to the quadratic term of superficial velocity.

$$
\frac{\Delta \mathrm{P}}{\mathrm{L}}=150 \frac{(1-\varepsilon)^{2}}{\varepsilon^{3}} \frac{\mu \mathrm{U}}{\mathrm{D}_{\mathrm{p}}^{2}}+1.75 \frac{1-\varepsilon}{\varepsilon^{3}} \frac{\rho \mathrm{U}^{2}}{\mathrm{D}_{\mathrm{p}}}
$$

Ergun (1952) combined the Carman (1937)Kozeny (1927) equation and Burke and Plummer (1928) in the form of the Forchheimer equation, is provided by Eq. (7).

$$
\mathrm{f}_{\mathrm{p}}=\frac{\Delta \mathrm{PD}_{\mathrm{p}}}{\rho L U^{2}} \frac{\varepsilon^{3}}{(1-\varepsilon)}
$$

Ergun equation can be rearranged in the form of friction factor, as show in Eq. (8).

$$
\mathrm{f}_{\mathrm{p}}=\frac{150}{\operatorname{Re}_{\mathrm{p}}}+1.75
$$

Similarly to the prediction model pressure drop is of the form of the Eq. (9). 
Ergun combines the Carman-Kozeny equation and Burke and Plummer in the form of Forchheimer equation. Macdonald used successfully Forchheimer equation and correlating the data separately for different porous beds in terms of various parameters including the surface roughness of the particle.

The velocity used in the theoretical analysis will be the same as in the experiment. The porosity is determined by the equation removed the chart of the experiment with different heights and corresponding weights.

\section{EXPERIMENTAL APPARATUS}

For the experimental analysis of the porous bed in the wind tunnel, the methodology used was to collect data of pressure drop, performing various tests taken for different lengths of beds and describe more clearly the process according to this variation.

To provide different height bed, a length of half the diameter of the PVC reactor $(0.075 \mathrm{~m})$ were assembled and separated only by overlapping steel grid. Each length was delimited by the grid, with a total of seven lengths according to the diameter of the reactor at a rate of $0.5 \mathrm{D}$.

The wind tunnel used is an open circuit, and straight-walled rectangular section of $0.30 \mathrm{~m} \times 0.30 \mathrm{~m}$ coupled to a PVC circular section of $0.15 \mathrm{~m}$ in diameter, in which were carried out data collection. These measurements are consistent with the bed of the gasifier so that they could make the analogy between the bed in the wind tunnel and at the downdraft gasifier.

The wind tunnel provides an uniform flow at the entrance of the test section, and for this work, the speed is varied according to frequency established on the frequency inverter and observing the corresponding reading velocity with the assistance of a pitot tube inserted into the flow at a distance of $1.95 \mathrm{~m}$ from the porous bed as shown in Fig. 4.

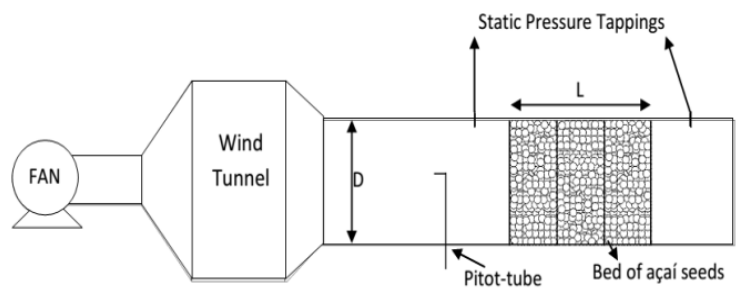

Figure 4. A sketch of the experimental test set-up.

The fixed porous bed used was composed of several nuclei of açaí seeds, arranged randomly, always obeying the limit of each section length with the assistance of steel grates of fine mesh, which did not allowed the mixture of the lengths and provided a good packed bed.

The açaí seeds were treated in a machine suitable for the removal of fibers so as to have the actual geometry of the cores.

\section{RESULTS AND DISCUSSION}

In experimental study carried out in the wind tunnel for different bed lengths of açaí seeds were collected in total 112,500 values of differential pressure and velocity to ensure repeatability in the experiment. After collecting, the values were treated to make it possible to have the mean values and standard deviations for the observed phenomenon.

The tests were supported with a FC487 Datalogger, which enabled to collect a very wide number of data for each measurement point in order to ensure a low uncertainty previously demonstrated by Student's t distribution. The data acquisition Micro gauge to the microcomputer was performed through a serial RS-232 and through the program FC487 Datalogger Version 2.1.0. The data were saved in worksheets in XLS Microsoft Excel software.

The values obtained in data collection of pressure drop in beds of acai seeds were treated for each bed length, and varying speeds, showing the behavior of the flow according to these variables. Then Ergun equation was applied.

The empirical Ergun equation makes it clear that the term of the average velocity can be represented by both the incident velocity in bed as the output velocity, Kaviany (1995). We chose to work with the values of speed before the bed, because in gasification experimental study the velocity is measured above the bed, so we could have a far more approximate average speed of the correlation that would be used in the technology of gasification.

The values obtained by the Ergun equation for pressure drop are shown in Fig. 5 with the experimental values. Analyzing the graph can be seen that the theoretical values are well above those measured experimentally for high Reynolds number.

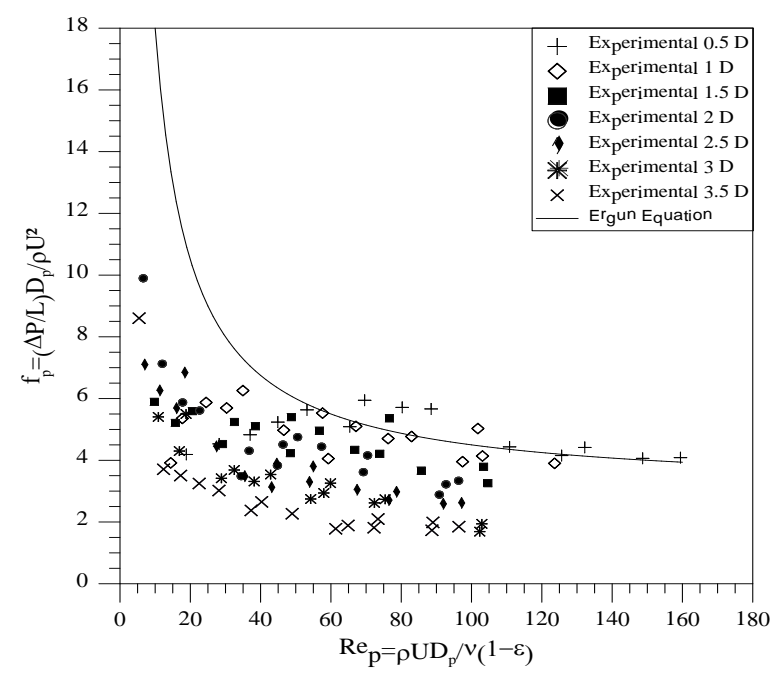

Figure 5. Comparison of experimental data and Ergun equation.

As is noted, the bed of açaí seeds serves an important role in the pressure drop in flow, is a 
phenomenon that cannot be neglected. It can be observed how the incident air flow is modified at the entrance of the fixed porous bed for a situation without the bed of açaí seeds, and get a broad overview of how the loss of load flow in porous bed behaves.

In the gasification case in which the fixed bed consumption occurs gradually, the decrease in the bed length would cause a too high difference in the process control. In the beginning of the gasification the pressure drop tends to be higher than that foreseen, so that a modification on the flow rate would be used to try to overcome the high pressure loss. This could undermine the gasification process.

\section{CONCLUSIONS}

The experimental study to determine the pressure drop in porous media of açaí seeds proceeded satisfactorily and met expectations for the operation and control in the downdraft gasifier process mentioned. According to the analysis using Ergun formulation in comparison to the experimental values obtained in laboratory it was observed a disagreement. As the Reynolds number increases the values for pressure drop are more distanced from the Ergun equation. The roughness effect of the açaí particle may have been a factor in the porosity that can promote the disagreement with the Ergun equation.

The length bed of $3.5 \mathrm{D}$ showed results lower than expected by the equation, which assumes that for this length the values were extrapolated, obtaining pressure loss smaller than expected. The pressure drop tends to exceed the values predicted by the Ergun equation. When the bed length is of $0.5 \mathrm{D}$ is practically impossible to use this formulation.

Quantitatively due to the error margin between 10 and $80 \%$ compared to the Ergun equation, it becomes apparent incompatibility of this equation for determining the pressure drop in downdraft gasifiers. Possible corrections in the equation must be discussed in future to apply in these cases.

The porosity determination of the fixed porous bed from açaí seeds proved to be quite satisfactory with a low uncertainty, the range of \pm 0.03 , and the expected values due to the large population sample of lumps.

\section{ACKNOWLEDGEMENTS}

The paper was conducted through funding from National Council of Scientific Development and Technology - CNPq - Brasil.

\section{REFERENCES}

Ahmed, N., and Sunada, D. K., 1969, Non Linear flow in Porous Media, Journal of Hydraulic Division, ASCE, Vol. 95, No. 6, pp. 1847-1857.
Burke, S. P., and Plummer, W. B., 1928, Gas Flow Through Packed Columns, Industrial and Engineering Chemistry, Vol. 20, pp. 1196-1200.

Carman, P. C., 1937, Fluid flow Through Packed Beds, Trans. IChemE, Vol. 15, pp. 150-166.

Churchill, S. W., 1988, Viscous Flow-The Practical Use of Theory, Butterworth Publishers.

Ergun, S., 1952, Fluid Flow Through Packed Columns, Chemical Engineering Progress, Vol. 48, No. 2, pp. 89-94.

Forchheimer, P., 1991, Wasserbewegung durch Boden, Zeitschrift des Vereines Deutscher Ingenieuer, 45th edition. (in German)

Kaviany, M., 1995, Principles of Heat Transfer in Porous Media, Second edition, Springer, NewYork.

Keyser, M. J., Conradie, M., Coertzen, M., and Van Dyk, J. C., 2006, Effect of Coal Particle Size Distribution on Packed Bed Pressure Drop and Gas Flow Distribution, Fuel, Vol. 85, pp. 1439-1445.

Kozeny, J., 1927, Uber Kapillare Leitung der Wasser in Boden, Sitzungs-ber, Akad Wiss. Wien, Vol. 136, pp. 271-306.

Leva, M., 1947, Pressured drop Through Packed Tubes, Chemical Engineering Progress, Vol. 43, pp. 549-554.

Li, L., and Ma, W., 2011, Experimental Study on the Effective Particle Diameter of a Packed Bed with Non-Spherical Particles, Transp Porous Med, Vol. 89, pp. 35-48.

Macdonald, I. F., El-Sayed, E. S., Mow, K., and Dullien, F. A. L., 1979, Flow through Porous Mediathe Ergun Equation Revisited, Industrial \& Engineering Chemistry Fundamentals, Vol. 18, No. 3, pp. 199-208.

Montillet, A., Akkari, E., and Comiti, J., 2007, About a Correlating Equation for Predicting Pressure Drops Through Packed Beds of Spheres in a Large Range of Reynolds Numbers, Chemical Engineering and Processing, Vol. 46, pp. 329-333.

Nield, D. A., and Bejan, A., 1992, Convection in Porous Media, Springer.

Niven, K. R., 2000, Physical Insight into the Ergun and Wen \& Yu Equations for Fluid Flow in Packed and Fluidised Beds, Chemical Engineering science, Vol. 57, pp. 527-534.

Ozahi, E., Gundogdu, M. Y., and Carpinlioglu, M. Ö., 2008, A Modification on Ergun's Correlation for Use in Cylindrical Packed Beds With Nonspherical Particles, Advanced Powder Technology, Vol. 18, pp. 369-381. 